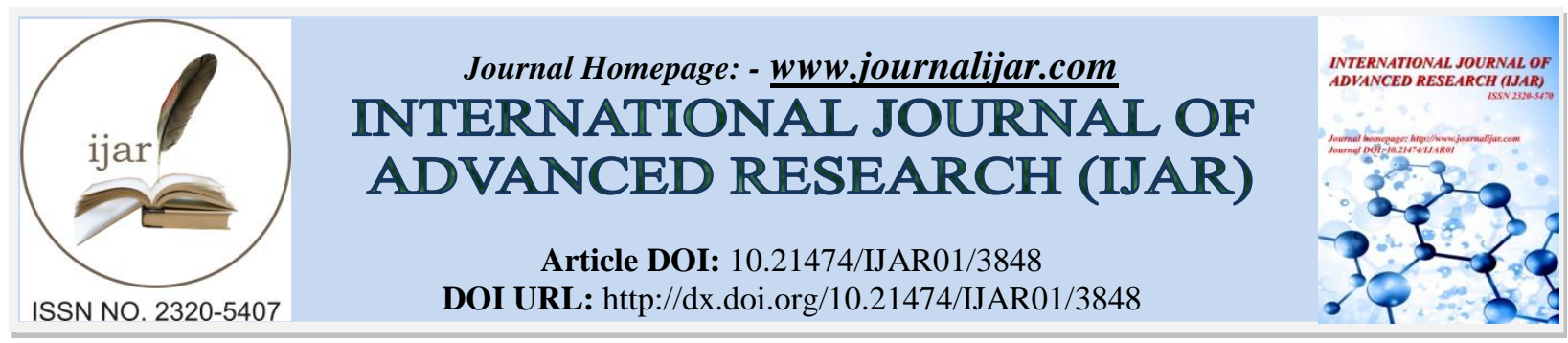

RESEARCH ARTICLE

\title{
ORAL LICHEN PLANUS; A CASE REPOT.
}

Dr. Sura Ali Ahmed Fuoad Albayati.

Associate Professor in Oral Medicine, Associate Dean College of Dentistry, Gulf Medical University- Ajman, UAE.

\section{Manuscript Info}

Manuscript History

Received: 05 February 2017

Final Accepted: 01 March 2017

Published: April 2017

\begin{abstract}
Oral lichen planus is a T-cell mediated chronic inflammation, raise due to infection, stress and has genetic background with a multifactorial pathogenesis. It is vary in symptoms and has many clinical presentations in the oral cavity,with or wiyhout the presence of the dermatological lesion. Reticular type diagnosed clinically, others needs histo-pathological confirmation. Management of lesion is individually, depend on the case with patient instruction.
\end{abstract}

Copy Right, IJAR, 2017,. All rights reserved.

\section{Introduction:-}

Lichen planus is a chronic immunological inflammation affecting skin, oral mucosa, scalp, nails, and other mucosa, it is non-infectious condition triggered by local and systemic triggers for cell mediated hypersensitivity reaction, as stress, viral infections, and epithelial antigens.[1]The disease is affecting 1-2\% of the population with female predilection and mostly in $5^{\text {th }}$ decades of life.[2]The apoptosis at the epithelial level is due to T CD8+ lymphocytes that result in the clinical manifestations, although some consider it of un known etiology and its pathogenesis is multi factorial. [3]

The clinical classifications of oral lichen planus shave been proposed by Silverman, who define three types: reticular, erosive and atrophic. The reticular form is most common asymptomatic or with few symptom, manifested as symmetrical white or grey streaks form a linear or reticular pattern (wickham striae) on an erythematous background of the buccal mucosa. The erosive and atrophic forms are symptomatic is with potential risk of malignant squamous cell carcinoma $0.4 \%$ to $2.5 \%$. [4,5]The atrophic form when it involve the whole gingiva it will be presented as desquamates gingivitis, combination of three types lesion may present in one site or involve different sites of oral mucosa.[6]

Symptoms associated with erosive and atrophic lichen planus may range from moderate to severe burning sensation with pain ,particularly during at spicy, worm food consumption, or may be roughness, on the other hand, the disease is not static, there is exacerbation and remission of symptoms.[7]

Based on history, clinical examination and biopsy to confirm the histo-pathplogical findings, the diagnosis is done. In addition, histo-pathological examination is to rule out malignancy or even epithelial dysplasia.

The goal of the treatment is to improve the lesion and accompanied symptoms, yet no complete remission or long remission could be achieved with any suggested treatment protocol. Topical or systemic corticosteroids are recommended with periodic follow up and monitoring the medication dosage.[1]

\section{Case Report:-}

A 39 Egyptian male, work as sale man, attend the outpatient clinic February 2017complaining from generalized whiting in the oral cavity, with mailed sourness especially during drinking worn drink and spicy food ,the white 
lesion appear 20 days before .The lesion didn't change is size ,shape or distribution in the oral cavity. The patient stop brushing of teeth science lesion appear.

Medical history is clear no history of new drug intake, no history of allergy.No family history of such lesion, the patient has been hospitalized 4 years back for intervertebral disc surgery. Patient quit smoking 2 years back.

On extra oral examination: no any skin lesion, and none of the facial lymph nodes are palpable.

On intra oral examination: poor oral hygiene stained teeth, calculus, gingival rescission in lower buccal and lingual sides, teeth are intact only 35 is missing. No prosthesis or faulty restoration.

On soft tissue examination: left side buccal mucosa at front opposing the premolars ,the mucosal lining of lower lip is with white lesion annular pattern, the right side buccal mucosa and the back left buccal mucosa is with irregular white lesion, buccal lesions are not extending to the vestibules, the palate and the floor of the mouth are clear. A longitudinal white lesion along the dorsal surface of the tongue on both sides of the central fissure is present. All white lesions cannot be raped off and on stretching did not disappear figures (1-4).

A swab and smear wear taken from buccal mucosa to rule out candida or any microbial infection, and the result came clear from bacterial and fungal infection. As a differential diagnosis, lichen planus was suggested which should be confirmed by biopsy. The patient was on kenaloge in orabase three times daily for 3 weeks, but no any change in the oral lesion was observed.

Biopsy was taken from lower lip measures $0.6 \mathrm{X} 0.4 \mathrm{X} 0.2 \mathrm{~cm}$, cut section was elliptical shape, grey white color, fixed in formalin and send for histopathology examination and diagnosis.

Instruction for good oral hygiene is given. Patient given a prescription of mouth wash without alcohol (the patient was fraid from brushing his teeth science the discovery of the lesion), Kenaloge orabase $1 \%$ to be used before meals and bed time, with prevention of spicy and worm food and drink.

The histo-pathological examination, reveals hyper-parakeratosis, squamatization of basal layer and the presence of civatte bodies, saw teeth rate ridges and band like lymphocyltic inflammatory infiltrate in the lamina properia. No signs of epithelial dysplasia. Same instructions were given to the patient for good oral hygiene and suppression of symptoms figure (5).
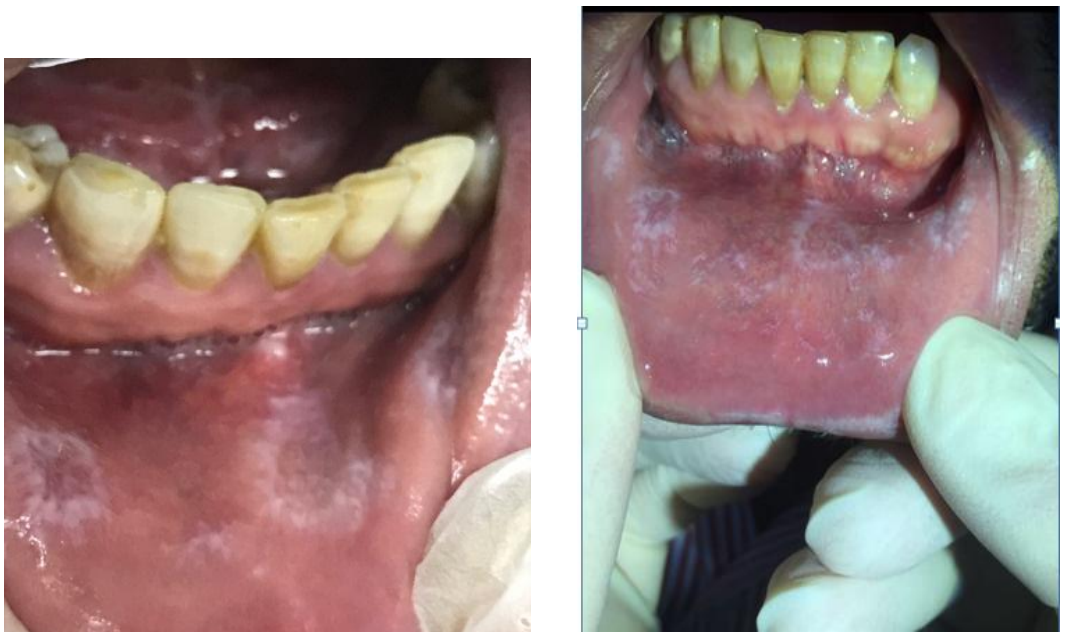

Figure 1:- Lower lip annular white lesion. 

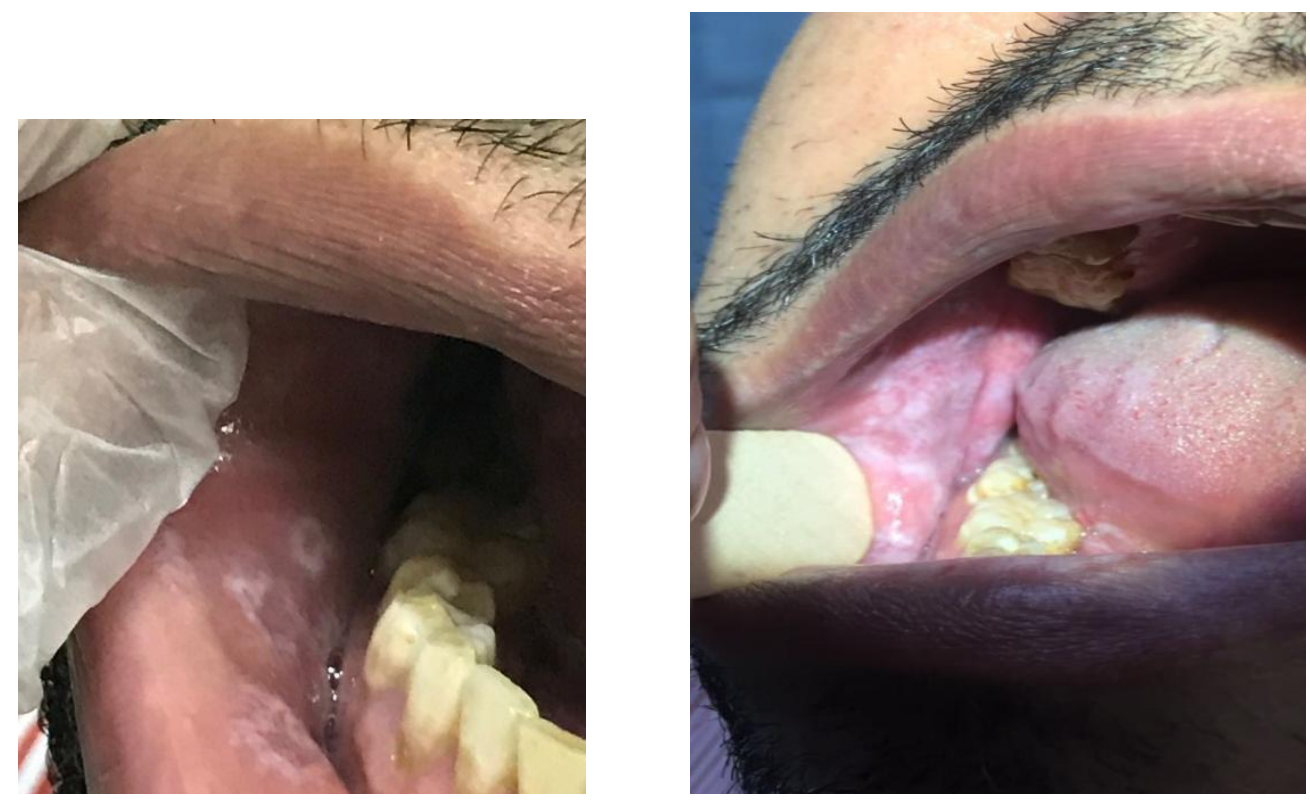

Figure 2:- Right cheek mucosa with annular and mix white lesion toward the vestibule and posteriorly.
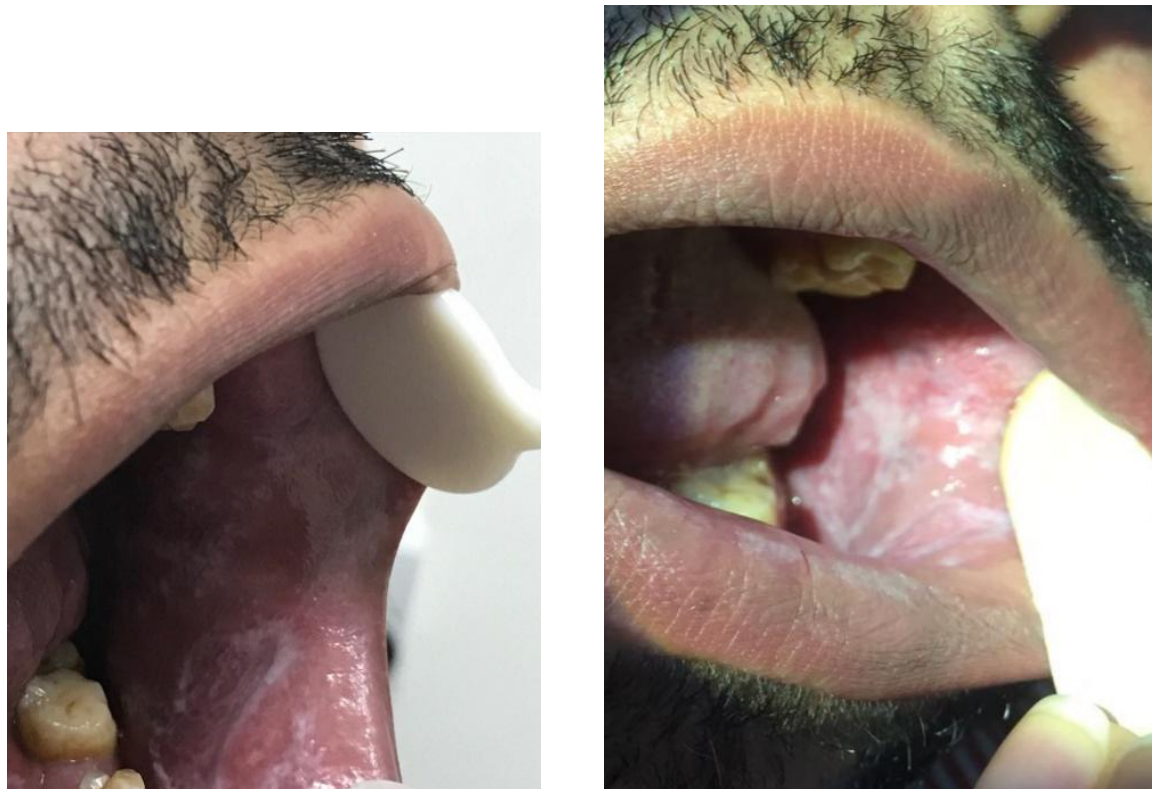

Figure 3:- Left cheek mucosa with mixed white lesion. 

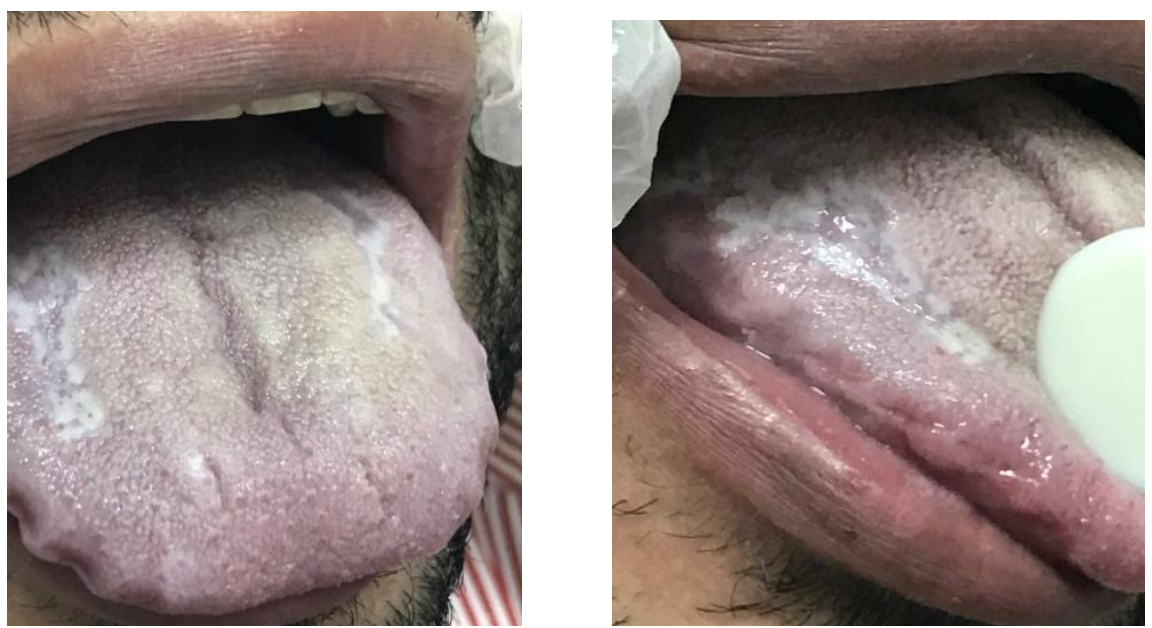

Figure 4:- Linear white lesion on dorsal surface of the tongue on both sides of mid fissure, indentation marks .

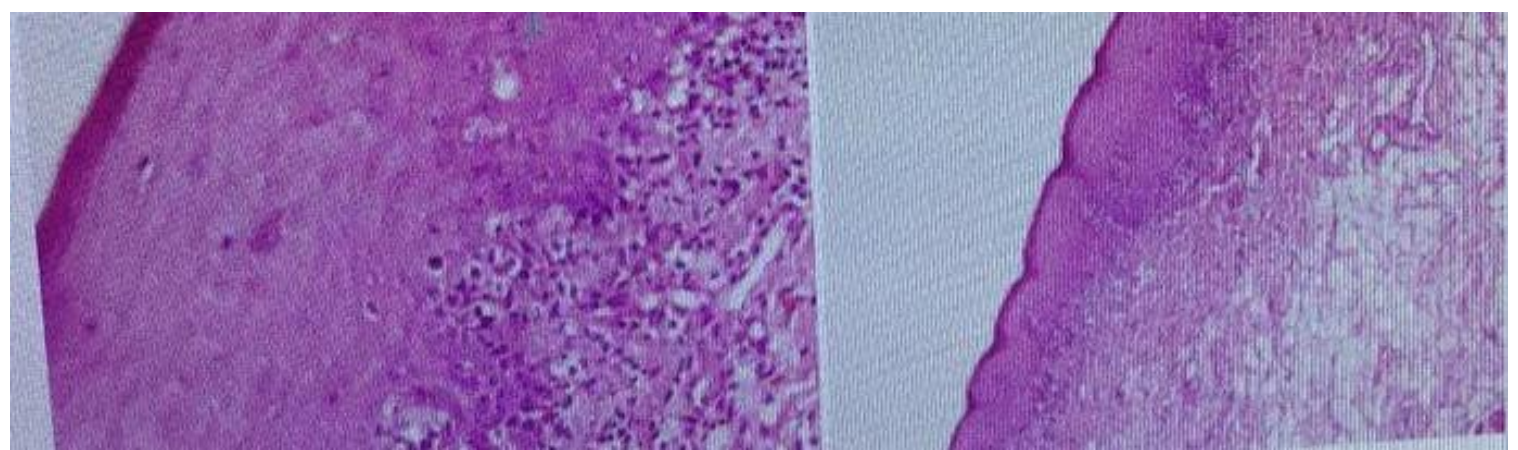

Figure 5:- Histopathology of lower lip mucosa with white lesion shows hyper parakeratosis squamatization of basal cell layer, saw teeth rate ridges and lymphocytic band infiltrate in sub mucosa.(Hematoxylin-eosin stain).

\section{Discussion:-}

Oral lichen planus is suggested to be an alteration of cell-mediated immunity that is triggered by exogenous or endogenous factors which end up with basal layer apoptosis of keratinocytes , on the other hand the deficiency in the immunosuppression mechanisms results in the chronicity of the disease.[8]

Clinical presentation orally, may be in reticular, atrophic, papular, erosive, bullous and erythematous forms, that may present simultaneously, with predominance of one form that could change over the time. Oral lesion is usually symmetrical, bi lateral .Commonest affected sites are: buccal mucosa, tongue gingiva and lip mucosa. Lesions of long evolution tend to become atrophic due to epithelial tissue correction. Atrophic lesion of the tongue result in depapillation leads to burning when contact with citrus and spicy foods.[7,9]

Lesion diagnosis performed by history, clinical examination and histo-pathological findings which is the acanthosis (hyper ortho, or para keratosis)if it is a keratotic white lesion, apoptosis of basal cell layer ,civatte bodies ,infiltration of lymphocytes in the lamina propria superficially, in addition to the saw teeth rate ridges.[7,10] Lesions resulting from contact with dental materials(restorations)will be asymmetrical and near the restoration, it represent lichenoid reaction, resolution will take place after change the restoration material.[11]Same could observed when new drug intake may result in liken planus like lesion that disappear after withdrawal of the new drug (lichenoid drug reaction).The use direct immune fluorescence assay is used to differentiate oral lichen planus from other mucocutanous condition for example lupus erythematosis, erythema multiformi and pemphigus .[12]

As oral lichen planus has an impact on daily life activities and patient psychology, the treatment goal is to minimize the symptoms and improve quality of patient's life. Prescription will be according to lesion extension and symptoms severity .For all patient's ,strict oral hygiene measures is essential ,recall follow up of the patient to monitor the drug dosage is also essential. Two to three times a day, topical corticosteroids - orabase paste, 
mouthwash is used .The main side effect is the candida infection, therefore, topical Nystatin is prophylactically used. In sever atrophic or ulcerative lesions low doses of systemic corticosteroids are use, increase the dose gradually till the symptoms disappear then gradually reduce the dose . [9]

\section{References:-}

1. Sura Ali Ahmed Fuoad Albayati, Tongue lichen planus:A case report and review.Int. J. Adv. Res. 4(10), 941946 .DOI URL:http://dx.doi.org/10.21474/IJAR01/1881.

2. Al-Hashimi I, Schifter M, Lockhart PB, Wray D, Brennan M, Migliorati CA, et al. Oral lichen planus and oral lichenoid lesions: diagnostic and therapeutic considerations. Oral Surg Oral Med Oral Pathol Oral Radiol Endod. 2007;103 Suppl:S25.e1-12.

3. 1. Ismail SB, Kumar SK, Zain RB. Oral lichen planus and lichenoid reactions: etiopathogenesis, diagnosis, management and malignant transformation. J Oral Sci. 2007;49:89-106.

4. Francisca Fernández-González, Rocío Vázquez-Álvarez, Dolores Reboiras-López, Pilar Gándara-Vila , Abel García-García , José-Manuel Gándara-Rey .Histopathological findings in oral lichen planus and their correlation with the clinical manifestations. Med Oral Patol Oral Cir Bucal. 2011 Aug 1;16 (5):e641-6.

5. Eisen D, Carrozzo M, Bagan Sebastian JV, Thongprasom K. Number V Oral lichen planus: clinical features and management. Oral Dis. 2005;11:338-49.

6. Eisen D, Carrozzo M, Bagan Sebastian JV, Thongprasom K. Number V Oral lichen planus: clinical features and management. Oral Dis. 2005;11:338-49.

7. Sura Ali Ahmed Fouad Al-Bayati. Oral Lichen planus: A clinical study of 123 patients attending an Oral Medicine Clinic, Baghdad University, Iraq. Gulf Medical Journal 2012;1(1):10-14.

8. Gorsky M, Epstein JB, Hasson-Kanfi H, Kaufman E. Smoking habits among patients diagnosed with oral lichen planus. Tobacco Induced Diseases. 2004;2:103

9. Marcello Menta Simonsen NicoI; Juliana Dumet FernandesII; Silvia Vanessa LourençoIII.

10. Oral lichen planus. An. Bras. Dermatol. 2011vol.86 no.4 . http://dx.doi.org/10.1590/S036505962011000400002.

11. Schlosser BJ. Lichen planus and lichenoid reactions of the oral mucosa. Dermatol Ther. 2010;23:251-67.

12. Laeijendecker R, Dekker SK, Burger PM, Mulder PG, Van Joost T, Neumann MH. Oral lichen planus and allergy to dental amalgam restorations. Arch Dermatol. 2004;140:1434-8.

13. Sampaio SAP, Rivitti EA. Erupções Papulo-pruriginosas. In: Sampaio SAP, Rivitti EA, eds. Dermatologia. 3 ed. São. Paulo: Artes Médicas; 2007. p. 277 - 99. 